\title{
Oblique Impact of Water Waves on Thin Porous Walls
}

\author{
H. J. Hsu'; L. H. Huang'; and P. C. Hsieh ${ }^{3}$
}

\begin{abstract}
There is a paradoxical phenomenon in earlier studies when the incoming water wave is parallel to a porous breakwater, the water wave permeates completely without regard to the largeness of the the porosity of the porous breakwater. For solving the problem of the water waves obliquely impacting upon the thin porous wall, a new boundary condition on the thin porous wall—which can remedy the above mentioned paradoxical phenomenon-is proposed based on the concept that the incident angle remains unchanged when the water wave permeates into the wall. According to this new boundary condition, an analytic solution of an oblique water wave impacting on a thin porous wall of any permeability is obtained. It is found that the above paradoxical phenomenon, as the water wave is parallel to a thin porous wall, disappears. And, as the incident angle approaches $90^{\circ}$, the reflection coefficient and the transmission coefficient reasonably converge to 1 and 0 , respectively, while on the contrary, those in the earlier investigations converge to 0 and 1 .
\end{abstract}

DOI: 10.1061/(ASCE)0733-9399(2005)131:7(721)

CE Database subject headings: Water waves; Thin wall structures; Porous media; Boundary conditions.

\section{Introduction}

The problem of water wave impact on a porous wall has been studied by many investigators. Sollitt and Cross (1972) formulated and solved the problem of a water wave passing through a porous medium but due to the complex wave numbers inside the porous medium the orthogonality of their infinite series solution is questionable. Madsen (1974) applied the method similar to Sollitt and Cross (1972) to analyze reflected and transmitted waves, but the same defect arises. Chwang (1983) used Taylor's (1956) concept of water flows through a porous screen to investigate the zero-thickness porous wavemaker problem. Later, Chwang and $\mathrm{Li}$ (1983), Chwang and Dong (1984), and Tyvand (1986) applied the same method in the analysis of a piston-type porous wavemaker and of the problem of water wave permeating through a porous screen. Note that in Chwang (1983), the inertial reactance of flow is neglected and the porous wall is assumed to have zero thickness.

Simplifying the theory of poroelasticity (see Biot 1962) by letting the skeleton of a porous medium be rigid and the fluid be incompressible, Huang (1991) obtained the governing equation of porous medium flow, similar to the one in Sollitt and Cross (1972). Chwang's (1983) study thus was extended to a finitethickness porous medium with the inertial reactance of the flow being preserved. Later, clarifying the boundary conditions for a

\footnotetext{
${ }^{1}$ Graduate Student, Dept. of Civil Engineering, National Taiwan Univ., Taipei, Taiwan, ROC.

${ }^{2}$ Professor, Dept. of Civil Engineering and Hydrotech Research Institute, National Taiwan Univ., Taipei, Taiwan, ROC.

${ }^{3}$ Associate Professor, Dept. of Soil and Water Conservation, National Chung Hsing Univ., Taipei, Taiwan, ROC.

Note. Associate Editor: Dewey H. Hodges. Discussion open until December 1, 2005. Separate discussions must be submitted for individual papers. To extend the closing date by one month, a written request must be filed with the ASCE Managing Editor. The manuscript for this paper was submitted for review and possible publication on September 23, 2002; approved on October 13, 2004. This paper is part of the Journal of Engineering Mechanics, Vol. 131, No. 7, July 1, 2005. (CASCE, ISSN 0733-9399/2005/7-721-732/\$25.00.
}

thin porous wall with both flow inertial reactance and viscous damping, Huang et al. (1993) analyzed the wave field of a thin porous wavemaker by a simplified analytical approach.

As for an oblique water wave impacting on a porous structure, Dalrymple et al. (1991) adopted an assumption that neglects the evanescent modes of the water wave near the interfaces of the porous wall to simplify their problem. It is found that when the incoming water wave is parallel to a porous breakwater, no matter how large the porosity of the porous wall is, the water wave permeates through completely. This is indeed questionable. Actually, to the writers' knowledge, this paradoxical phenomenon can be found in all the other earlier studies of oblique waves acting on porous structures.

For the purpose of eliminating the above mentioned paradoxical phenomenon, we attempt to derive a new boundary condition-for the thin porous wall impacted by an oblique wave-based on the concept that the wave direction remains unchanged as it permeates into a thin porous wall in the present study. According to this new boundary condition, an analytic solution of an oblique water wave impacting on a thin porous wall of any permeability is obtained. It is found that the above paradoxical phenomenon as the water wave is parallel to a thin porous wall disappears. And as the incident angle approaches $90^{\circ}$, the reflection coefficient and the transmission coefficient reasonably converge to 1 and 0 , respectively, while, on the contrary, those in the earlier researches converge to 0 and 1 .

\section{Formulation of Oblique Wave Field}

\section{Governing Equations}

Homogeneous water wave is assumed to be inviscid, irrotational, and incompressible; and the fluid domains of the present study are shown in Fig. 1.

For Regions (1) and (2), we have

$$
\underline{V}_{j}=\nabla \Phi_{j}, \quad j=1,2
$$

where $\underline{V}_{j}$ and $\Phi_{j}=$ velocity vectors and velocity potentials of fluid, respectively; and the subscript $j$ denotes the flow regions (see Fig. 


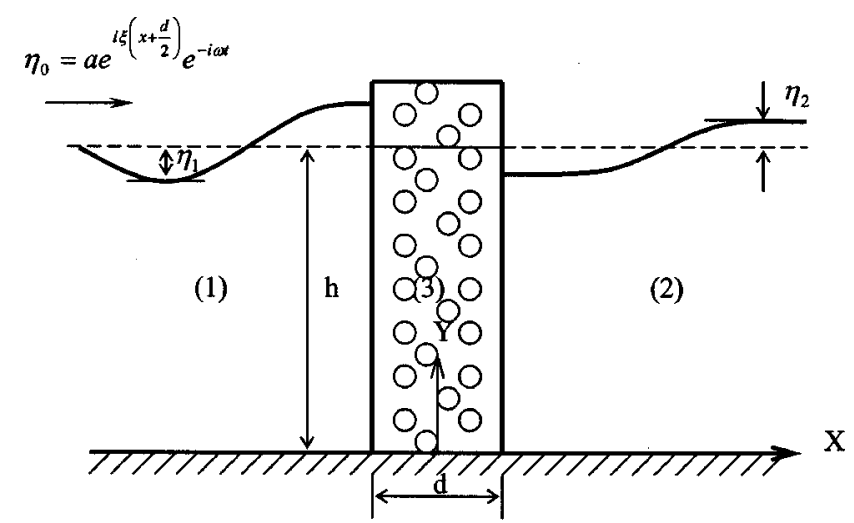

(a)

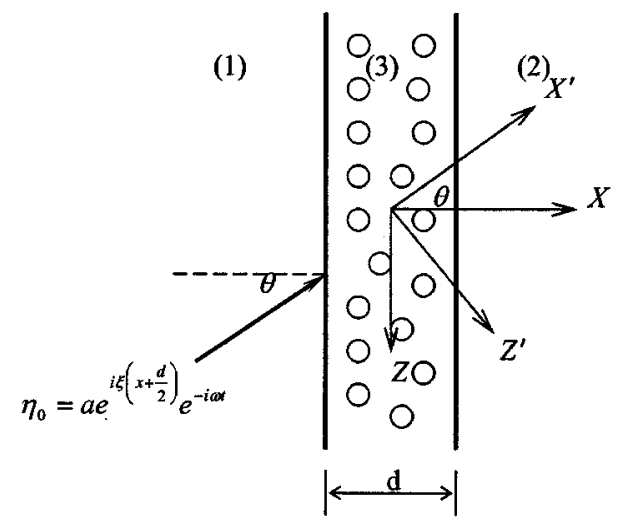

(b)

Fig. 1. Schematic diagram of water wave impacting on a porous wall: (a) side view; and (b) top view

1). And, the continuity equation and linear momentum equation are

$$
\begin{gathered}
\nabla \cdot \underline{V}_{j}=0, \quad j=1,2 \\
\rho \frac{\partial \underline{V}_{j}}{\partial t}=-\nabla p_{j}+\rho \underline{g}, \quad j=1,2
\end{gathered}
$$

By substituting Eq. (1) into Eq. (2), Eq. (2) becomes

$$
\nabla^{2} \Phi_{j}=0, \quad j=1,2
$$

Then, by substituting Eq. (1) into Eq. (3) and integrating the latter with respect to $y$, Eq. (3) becomes

$$
p_{j}=-\rho \frac{\partial \Phi_{j}}{\partial t}-\rho g(y-h), \quad j=1,2
$$

when it is not on the surface of seepage (e.g., see Muskat 1946); and

$$
p_{j}=0, \quad j=1,2
$$

when it is on the surface of seepage, where $p_{j}=$ pressure of the fluid; and $\rho=$ density of the fluid.

For Region (3) that is within the porous wall, we have

$$
\underline{V}_{3}=\nabla \Phi_{3}
$$

where $\underline{V}_{3}$ and $\Phi_{3}=$ velocity vector and velocity potential of fluid inside the porous wall, respectively (see Fig. 1). And, referring to
Huang (1991), the continuity equation and linear momentum equation are

$$
\begin{gathered}
\nabla \cdot \underline{V}_{3}=0 \\
\rho \frac{\partial \underline{V}_{3}}{\partial t}=-\nabla p_{3}+\rho \underline{g}-\frac{\mu n_{0}}{k} \underline{V}_{3}
\end{gathered}
$$

where $\mu=$ dynamic viscosity of fluid; $n_{0}$ and $k=$ porosity and specific permeability of the porous wall, respectively. By substituting Eq. (6) into Eq. (7), Eq. (7) becomes

$$
\nabla^{2} \Phi_{3}=0
$$

Then by substituting Eq. (6) into Eq. (8) and integrating the latter with respect to $y$, Eq. (8) becomes

$$
p_{3}=-\rho \frac{\partial \Phi_{3}}{\partial t}-\rho g(y-h)-\frac{\mu n_{0}}{k} \Phi_{3}
$$

when it is not on the surface of seepage; and

$$
p_{3}=0
$$

when it is on the surface of seepage.

\section{Boundary Conditions}

On the bottom (i.e., $y=0$ ), the normal velocity equals to zero, i.e., we get

$$
\frac{\partial \Phi_{j}}{\partial y}=0, \quad j=1,2,3
$$

On the free surface of fluid, $y=h$ and $-\infty<x<-d / 2$ for $\mathrm{Re}$ gion (1) and $d / 2<x<\infty$ for Region (2), we have the conventional kinematic and dynamic boundary conditions as

$$
\frac{\partial \Phi_{j}}{d y}=\frac{\partial \eta_{j}}{\partial t}, \quad j=1,2
$$

$$
\frac{\partial \Phi_{j}}{\partial t}+g \eta_{j}=0, \quad j=1,2
$$

where $\eta_{j}=$ vertical deviation of water surface from the mean-free surface. By combining Eqs. (12) and (13), we get

$$
\frac{\partial^{2} \Phi_{j}}{\partial t^{2}}+g \frac{\partial \Phi_{j}}{\partial y}=0, \quad j=1,2
$$

On the free surface of fluid inside the porous wall, $y=h$ and $-d / 2<x<d / 2$, the kinematic and dynamic boundary conditions are

$$
\begin{gathered}
\frac{\partial \Phi_{3}}{\partial y}=n_{0} \frac{\partial \eta_{3}}{\partial t} \\
\frac{\partial \Phi_{3}}{\partial t}+\frac{\mu n_{0}}{\rho k} \Phi_{3}+g \eta_{3}=0
\end{gathered}
$$

By combining Eq. (15) with Eq. (16), we get

$$
\frac{\partial^{2} \Phi_{3}}{\partial t^{2}}+\frac{\mu n_{0}}{\rho k} \frac{\partial \Phi_{3}}{\partial t}+\frac{g}{n_{0}} \frac{\partial \Phi_{3}}{\partial y}=0
$$

At the far field, $0<y<h$ and $x \rightarrow \pm \infty$, the radiation boundary condition is

$$
\lim _{x \rightarrow \pm \infty} \Phi_{j} \rightarrow \text { outgoing or } 0 \quad j=1,2
$$


On the interfaces of the porous wall and the fluid, $0<y<h$ and $x= \pm d / 2$, the continuities of mass flux and pressure are considered. From Eqs. (1) and (6), we have

$$
\frac{\partial \Phi_{j}}{\partial x}=n_{0} \frac{\partial \Phi_{3}}{\partial x}, \quad j=1,2
$$

and according to Eqs. $(5 a),(5 b),(10 a)$, and $(10 b)$ with the surface of seepage effect being ignored for convenience, it is obtained that

$$
\frac{\partial \Phi_{j}}{\partial t}=\frac{\partial \Phi_{3}}{\partial t}+\frac{\mu n_{0}}{\rho k} \Phi_{3}, \quad j=1,2
$$

\section{Boundary Value Problem}

Since the present study focuses on the problem of a periodic linear water wave, we may introduce a time factor $e^{-i \omega t}$ into the time-dependent variables, where $\omega$ is the angular frequency of water wave. And according to Snell's law, in order to satisfy the matching conditions at each vertical interface, we can write

$$
\Phi_{j}(x, y, z, t)=\mathrm{R}\left\lfloor\phi_{j}(x, y) e^{i\left(k_{0} \sin \theta_{z}-\omega t\right)}\right\rfloor, \quad j=1,2,3
$$

so that the $z$ variation of the solution in each region must be the same. In addition, a flow-efficiency parameter is defined as

$$
R=\frac{\text { inertial reaction force }}{\text { resistant force }}=\frac{\rho \omega k}{\mu n_{0}}
$$

which represents the capability for the fluid to flow through the porous medium (see Huang 1991). After getting rid of $e^{-i \omega t}$, the time-independent boundary value problem can be constructed by governing Eqs. (4) and (9) with boundary conditions (11), (14), and (17)-(20).

\section{Solution}

\section{Boundary Condition on Thin Porous Wall}

When the porous wall is extremely thin (i.e., $d$ in Fig. 1 is small), only the flow fields of Regions (1) and (2) need to be considered, i.e., we do not need to solve governing Eq. (9) with boundary conditions (11) for $j=3$, (17), (19), and (20). Therefore, the original boundary conditions (19) and (20) on the interfaces $(x$ $= \pm d / 2$ ) should be rectified and combined to obtain a new boundary condition that fits the thin porous wall and is independent of $\phi_{3}$.

We will use Sahoo et al. (2000) as an example to introduce the conventional way in dealing with the problem of an oblique wave's impact on a thin porous wall. Like other earlier studies, the boundary condition on the thin porous wall given in Sahoo et al. (2000) is obtained under the assumption that, regardless of the incident angles, the wave always permeates into the porous wall in normal direction (see Fig. 2). For this inference, even if the incoming water wave in Region (1) is parallel to the porous wall (i.e., $\theta=90^{\circ}$ ), there still exists a normal wave inside the porous wall which then permeates completely into Region (2). This phenomenon is indeed paradoxical and is obviously against our physical expectation when the viscous effect of the water wave is so little and hence negligible. However, as the present writers know, this paradoxical result of $\theta=90^{\circ}$ happens in all the earlier studies of relative problems; e.g. Darymple et al. (1991), Sahoo et al. (2000), etc.

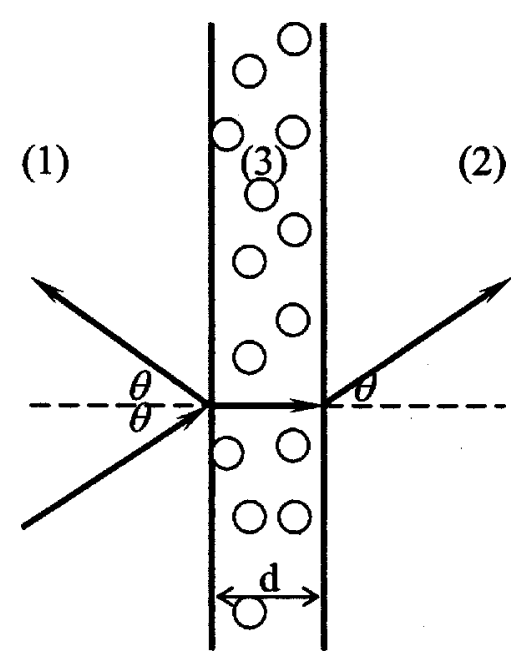

Fig. 2. Schematic diagram of oblique water wave impacting on a thin porous wall [based on data from Sahoo et al. (2000)]

After many thoughts, we find that since the wavelength is much longer than the thickness of the porous wall, there should be no diffraction. And because the water that transports the wave inside the porous wall is still the same as those in Regions (1) and (2), no refraction occurs either. Hence, a new boundary condition on the thin porous wall-which can remedy the above mentioned paradoxical phenomenon as $\theta=90^{\circ}$-is proposed based on the concept that the incident angle $\theta$ remains unchanged when the water wave permeates into Region (3) as shown in Fig. 3.

Before the new boundary condition is derived, we may see from Fig. 3 that because the flow in Region (3) follows Darcy's law of porous media flow (i.e., the velocity vector is linearly proportional to the pressure gradient inside the porous wall), when the incoming water wave is parallel to the thin porous wall (i.e., $\theta=90^{\circ}$ ), as long as the viscous effect of fluid on the wall is negligible, the water wave will no longer penetrate the porous wall and appearing in Region (2).

We now start to derive our new boundary condition on the thin porous wall. For the oblique incoming wave, as shown in Fig. 3, the flow is in the direction of $x^{\prime}$ axis and passes through $\underline{x}_{1}\left(-d / 2, y, z_{1}\right)$ and $\underline{x}_{2}\left(d / 2, y, z_{2}\right)$ on the wall. Therefore, it is necessary to take velocity vectors at $\underline{x}=\underline{x}_{1}$ and $\underline{x}=\underline{x}_{2}$. And, according to Huang et al. (1993), it is quite acceptable that the velocities are

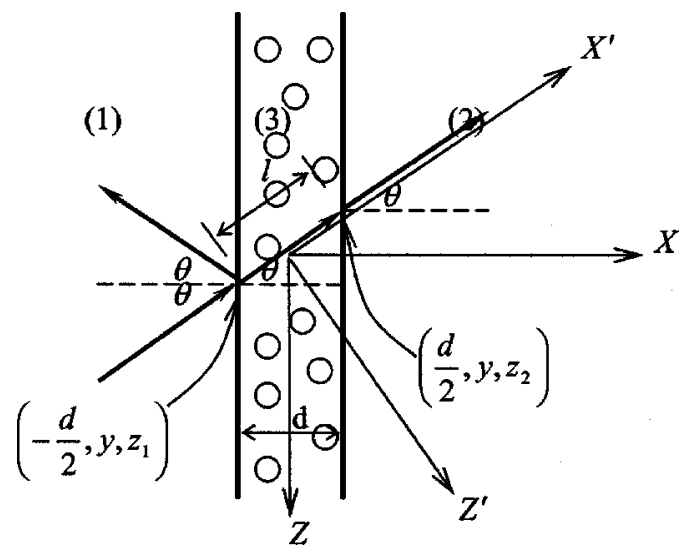

Fig. 3. Schematic diagram of oblique water wave impacting on a thin porous wall in the present study 
identical from the entrance to the exit along a streamline inside the thin porous wall. Then, referring to Huang et al. (1993), we get

$$
\left.\frac{\partial \phi_{3}}{\partial x^{\prime}}\right|_{\left(-d / 2, y, z_{1}\right)}=\left.\frac{\partial \phi_{3}}{\partial x^{\prime}}\right|_{\left(d / 2, y, z_{2}\right)}=\frac{1}{l}\left(\left.\phi_{3}\right|_{\left(d / 2, y, z_{2}\right)}-\left.\phi_{3}\right|_{\left(-d / 2, y, z_{1}\right)}\right)
$$

Substituting $\partial \phi_{3} / \partial x^{\prime}=(1 / \cos \theta)\left(\partial \phi_{3} / \partial x\right)$ and $l=d / \cos \theta$ into Eq. (23), it is obtained that

$$
\begin{aligned}
\left.\frac{\partial \phi_{3}}{\partial x}\right|_{\left[-(d / 2), y, z_{1}\right]} & =\left.\frac{\partial \phi_{3}}{\partial x}\right|_{\left(d / 2, y, z_{2}\right)} \\
& =\frac{1}{d}\left(\left.\phi_{3}\right|_{\left(d / 2, y, z_{2}\right)}-\left.\phi_{3}\right|_{\left[-(d / 2), y, z_{1}\right]}\right) \cos ^{2} \theta
\end{aligned}
$$

On the other hand, referring to the continuous mass flux condition and continuous pressure condition on the interfaces (i.e., at $\underline{x}=\underline{x}_{1}$ and $\underline{x}=\underline{x}_{2}$ ), Eq. (19) can be rewritten as

$$
\begin{aligned}
& \left.\frac{\partial \phi_{1}}{\partial x}\right|_{\left[-(d / 2), y, z_{1}\right]}=\left.n_{0} \frac{\partial \phi_{3}}{\partial x}\right|_{\left[-(d / 2), y, z_{1}\right]} \\
& \left.\frac{\partial \phi_{2}}{\partial x}\right|_{\left(d / 2, y, z_{2}\right)}=\left.n_{0} \frac{\partial \phi_{3}}{\partial x}\right|_{\left(d / 2, y, z_{2}\right)}
\end{aligned}
$$

and Eq. (20) can be expressed as

$$
\begin{aligned}
\left.i R \phi_{1}\right|_{\left[-(d / 2), y, z_{1}\right]} & =\left.(i R-1) \phi_{3}\right|_{\left[-(d / 2), y, z_{1}\right]} \\
\left.i R \phi_{2}\right|_{\left(d / 2, y, z_{2}\right)} & =\left.(i R-1) \phi_{3}\right|_{\left(d / 2, y, z_{2}\right)}
\end{aligned}
$$

Eq. (28) minus Eq. (27) gives

$$
\begin{aligned}
& i R\left(\left.\phi_{2}\right|_{\left(d / 2, y, z_{2}\right)}-\left.\phi_{1}\right|_{\left[-(d / 2), y, z_{1}\right]}\right) \\
& \quad=(i R-1)\left(\left.\phi_{3}\right|_{\left(d / 2, y, z_{2}\right)}-\left.\phi_{3}\right|_{\left[-(d / 2), y, z_{1}\right]}\right)
\end{aligned}
$$

Now, substituting Eqs. (25), (26), and (29) into Eq. (24) and getting rid of $\phi_{3}$, the final form of boundary conditions on the interfaces are obtained as

$$
\begin{aligned}
\left.\frac{\partial \phi_{1}}{\partial x}\right|_{\left[-(d / 2), y, z_{1}\right]} & =\left.\frac{\partial \phi_{2}}{\partial x}\right|_{\left(d / 2, y, z_{2}\right)} \\
& =\frac{n_{0}}{d} \frac{i R}{1-i R}\left(\left.\phi_{1}\right|_{\left[-(d / 2), y, z_{1}\right]}-\left.\phi_{2}\right|_{\left(d / 2, y, z_{2}\right)}\right) \cos ^{2} \theta
\end{aligned}
$$

Eq. (30) is the boundary condition suitable for the thin porous wall impacted by the oblique water wave. It is obviously observed from Eqs. (25), (26), and (30) that when $\theta=90^{\circ}$, there will be no normal velocity on each side of the porous wall, which satisfies Darcy's law and indeed agrees to our physical expectation.

From Fig. 3, we find $z=z_{1}-(d / 2) \tan \theta=z_{2}+(d / 2) \tan \theta$. Hence, Eq. (30) can be written as

$$
\begin{aligned}
\left.\frac{\partial \phi_{1}}{\partial x}\right|_{[-(d / 2), y, z]} & =\left.\frac{\partial \phi_{2}}{\partial x}\right|_{(d / 2, y, z)} \\
& =\frac{n_{0}}{d} \frac{i R}{1-i R}\left(\left.\phi_{1}\right|_{[-(d / 2), y, z]}-\left.\phi_{2}\right|_{(d / 2, y, z)}\right) \cos ^{2} \theta
\end{aligned}
$$

as a good approximation when $d$ is extremely small. Eq. (30a) is the boundary condition that we are going to use. The boundary condition on the thin porous wall which is used in Sahoo et al. (2000) is similar to Eq. (30a) but without $\cos ^{2} \theta$.

\section{Analytical Solution}

By applying Eqs. (21) and (22) into the governing Eq. (4) and boundary conditions (11), (14), (18), and (30a), the analytical solution is easily obtained as

$$
\begin{aligned}
& \phi_{1}(x, y, z)=\phi_{0}\left\{e^{i \xi[x+(d / 2)]}+A_{0} e^{-i \xi(x+(d / 2))}\right\} \cosh \left(k_{0} y\right) e^{i \zeta z} \\
& \phi_{2}(x, y, z)=\phi_{0}\left\{e^{i \xi[x-(d / 2)]}-A_{0} e^{i \xi[x-(d / 2)]}\right\} \cosh \left(k_{0} y\right) e^{i \zeta z}
\end{aligned}
$$

with

$$
\begin{gathered}
\phi_{0}=-\frac{i a g}{\omega \cosh \left(k_{0} h\right)} \\
A_{0}=\frac{\xi d(1-i R)}{\xi d(1-i R)+2 n_{0} R \cos ^{2} \theta}
\end{gathered}
$$

where $\xi=k_{0} \cos \theta, \zeta=k_{0} \sin \theta$ and $k_{0}$ satisfies the dispersion relation

$$
\omega^{2}-g k_{0} \tanh \left(k_{0} h\right)=0
$$

It is clearly found that when $\theta=90^{\circ}$,

$$
\begin{gathered}
\phi_{1}(x, y, z)=2 \phi_{0} \cosh \left(k_{0} y\right) e^{i k_{0} z} \\
\phi_{2}(x, y, z)=0
\end{gathered}
$$

Eq. (37), again, indicates that when the incoming water wave is parallel to the porous wall, Region (2) remains quiescent and the wave field does not appear. Hence, the paradox which occurs when the parallel water wave acting on the thin porous wall is eliminated.

\section{Physical Properties}

Since the $z$ variation of analytical solutions are the same, we may study all the physical properties in the condition that $z=0$ for representation.

By Eqs. (5a) and (5b), the nondimensional perturbed pressures on the interfaces $(x= \pm d / 2)$ are obtained as

$$
\left.\frac{p_{1}}{\rho \omega^{2} a h}\right|_{x=-(d / 2)}=A_{p_{1}} \cos \omega t+B_{p_{1}} \sin \omega t=D_{p_{1}} \cos \left(\theta_{p_{1}}-\omega t\right)
$$

where

$$
\begin{gathered}
A_{p_{1}}=-\frac{1}{\omega a h} \operatorname{Im}\left(\phi_{0}\right)\left[1+\mathrm{R}\left(A_{0}\right)\right] \cosh \left(k_{0} y\right) \\
B_{p_{1}}=-\frac{1}{\omega a h} \operatorname{Im}\left(\phi_{0}\right) \operatorname{Im}\left(A_{0}\right) \cosh \left(k_{0} y\right) \\
D_{p_{1}}=\sqrt{A_{p_{1}}^{2}+B_{p_{1}}^{2}} \\
\theta_{p_{1}}=\tan ^{-1}\left(\frac{B_{p_{1}}}{A_{p_{1}}}\right)
\end{gathered}
$$




$$
\left.\frac{p_{2}}{\rho \omega^{2} a h}\right|_{x=d / 2}=A_{p_{2}} \cos \omega t+B_{p_{2}} \sin \omega t=D_{p_{2}} \cos \left(\theta_{p_{2}}-\omega t\right)
$$

where

$$
\begin{gathered}
A_{p_{2}}=-\frac{1}{\omega a h} \operatorname{Im}\left(\phi_{0}\right)\left[1-\mathrm{R}\left(A_{0}\right)\right] \cosh \left(k_{0} y\right) \\
B_{p_{2}}=\frac{1}{\omega a h} \operatorname{Im}\left(\phi_{0}\right) \operatorname{Im}\left(A_{0}\right) \cosh \left(k_{0} y\right) \\
D_{p_{2}}=\sqrt{A_{p_{2}}^{2}+B_{p_{2}}^{2}} \\
\theta_{p_{2}}=\tan ^{-1}\left(\frac{B_{p_{2}}}{A_{p_{2}}}\right)
\end{gathered}
$$

where $\mathrm{R}$ and $\mathrm{Im}$ indicate real and imaginary parts, respectively.

By Eq. (1), the nondimensional normal velocities on the interfaces $(x= \pm d / 2)$ are obtained as

$$
\left.\frac{U_{1}}{-a \omega}\right|_{x=-(d / 2)}=A_{U_{1}} \cos \omega t+B_{U_{1}} \sin \omega t=D_{U_{1}} \cos \left(\theta_{U_{1}}-\omega t\right)
$$

where

$$
\begin{gathered}
A_{U_{1}}=\frac{1}{a \omega} \xi \operatorname{Im}\left(\phi_{0}\right)\left[1-\mathrm{R}\left(A_{0}\right)\right] \cosh \left(k_{0} y\right) \\
B_{U_{1}}=-\frac{1}{a \omega} \xi \operatorname{Im}\left(\phi_{0}\right) \operatorname{Im}\left(A_{0}\right) \cosh \left(k_{0} y\right) \\
D_{U_{1}}=\sqrt{A_{U_{1}}^{2}+B_{U_{1}}^{2}} \\
\theta_{U_{1}}=\tan ^{-1}\left(\frac{B_{U_{1}}}{A_{U_{1}}}\right) \\
\left.\frac{U_{2}}{-a \omega}\right|_{x=d / 2}=A_{U_{2}} \cos \omega t+B_{U_{2}} \sin \omega t=D_{U_{2}} \cos \left(\theta_{U_{2}}-\omega t\right)
\end{gathered}
$$

where

$$
\begin{gathered}
A_{U_{2}}=\frac{1}{a \omega} \xi \operatorname{Im}\left(\phi_{0}\right)\left[1-\mathrm{R}\left(A_{0}\right)\right] \cosh \left(k_{0} y\right) \\
B_{U_{2}}=-\frac{1}{a \omega} \xi \operatorname{Im}\left(\phi_{0}\right) \operatorname{Im}\left(A_{0}\right) \cosh \left(k_{0} y\right) \\
D_{U_{2}}=\sqrt{A_{U_{2}}^{2}+B_{U_{2}}^{2}} \\
\theta_{U_{2}}=\tan ^{-1}\left(\frac{B_{U_{2}}}{A_{U_{2}}}\right)
\end{gathered}
$$

Similarly, by Eq. (1), the nondimensional tangential velocities on the interfaces $(x= \pm d / 2)$ are obtained as

$$
\left.\frac{V_{1}}{-a \omega}\right|_{x=-(d / 2)}=A_{V_{1}} \cos \omega t+B_{V_{1}} \sin \omega t=D_{V_{1}} \cos \left(\theta_{V_{1}}-\omega t\right)
$$

where

$$
\begin{gathered}
A_{V_{1}}=\frac{1}{a \omega} k_{0} \operatorname{Im}\left(\phi_{0}\right) \operatorname{Im}\left(A_{0}\right) \sinh \left(k_{0} y\right) \\
B_{V_{1}}=-\frac{1}{a \omega} k_{0} \operatorname{Im}\left(\phi_{0}\right)\left[1+\mathrm{R}\left(A_{0}\right)\right] \sinh \left(k_{0} y\right) \\
D_{V_{1}}=\sqrt{A_{V_{1}}^{2}+B_{V_{1}}^{2}} \\
\theta_{V_{1}}=\tan ^{-1}\left(\frac{B_{V_{1}}}{A_{V_{1}}}\right)
\end{gathered}
$$

$$
\left.\frac{V_{2}}{-a \omega}\right|_{x=d / 2}=A_{V_{2}} \cos \omega t+B_{V_{2}} \sin \omega t=D_{V_{2}} \cos \left(\theta_{V_{2}}-\omega t\right)
$$

where

$$
A_{V_{2}}=-\frac{1}{a \omega} k_{0} \operatorname{Im}\left(\phi_{0}\right) \operatorname{Im}\left(A_{0}\right) \sinh \left(k_{0} y\right)
$$

$$
B_{V_{2}}=-\frac{1}{a \omega} k_{0} \operatorname{Im}\left(\phi_{0}\right)\left[1-\mathrm{R}\left(A_{0}\right)\right] \sinh \left(k_{0} y\right)
$$

$$
\begin{aligned}
& D_{V_{2}}=\sqrt{A_{V_{2}}^{2}+B_{V_{2}}^{2}} \\
& \theta_{V_{2}}=\tan ^{-1}\left(\frac{B_{V_{2}}}{A_{V_{2}}}\right)
\end{aligned}
$$

By Eq. (13), the wave profile which takes off the incoming wave $\eta_{0}$ in Region (1) and wave profile in Region (2) are obtained as

$$
\begin{aligned}
\left.\frac{\eta_{1}}{a}\right|_{y=h}= & \mathrm{R}\left(A_{0}\right) \cos \left(\xi\left(x+\frac{d}{2}\right)+\omega t\right) \\
& +\operatorname{Im}\left(A_{0}\right) \sin \left(\xi\left(x+\frac{d}{2}\right)+\omega t\right) \\
\left.\frac{\eta_{2}}{a}\right|_{y=h}= & \cos \left(\xi\left(x-\frac{d}{2}\right)-\omega t\right)-\mathrm{R}\left(A_{0}\right) \cos \left(\xi\left(x-\frac{d}{2}\right)-\omega t\right) \\
+ & \operatorname{Im}\left(A_{0}\right) \sin \left(\xi\left(x-\frac{d}{2}\right)-\omega t\right)
\end{aligned}
$$

The reflection coefficient and transmission coefficient of the wave field are defined as

$$
\begin{gathered}
C_{r}=\left|\frac{\eta_{1}}{a}\right|^{2}=\mathrm{R}\left(A_{0}\right)^{2}+\operatorname{Im}\left(A_{0}\right)^{2}, \\
C_{t}=\left|\frac{\eta_{2}}{a}\right|^{2}=\left[1-\mathrm{R}\left(A_{0}\right)\right]^{2}+\operatorname{Im}\left(A_{0}\right)^{2}
\end{gathered}
$$



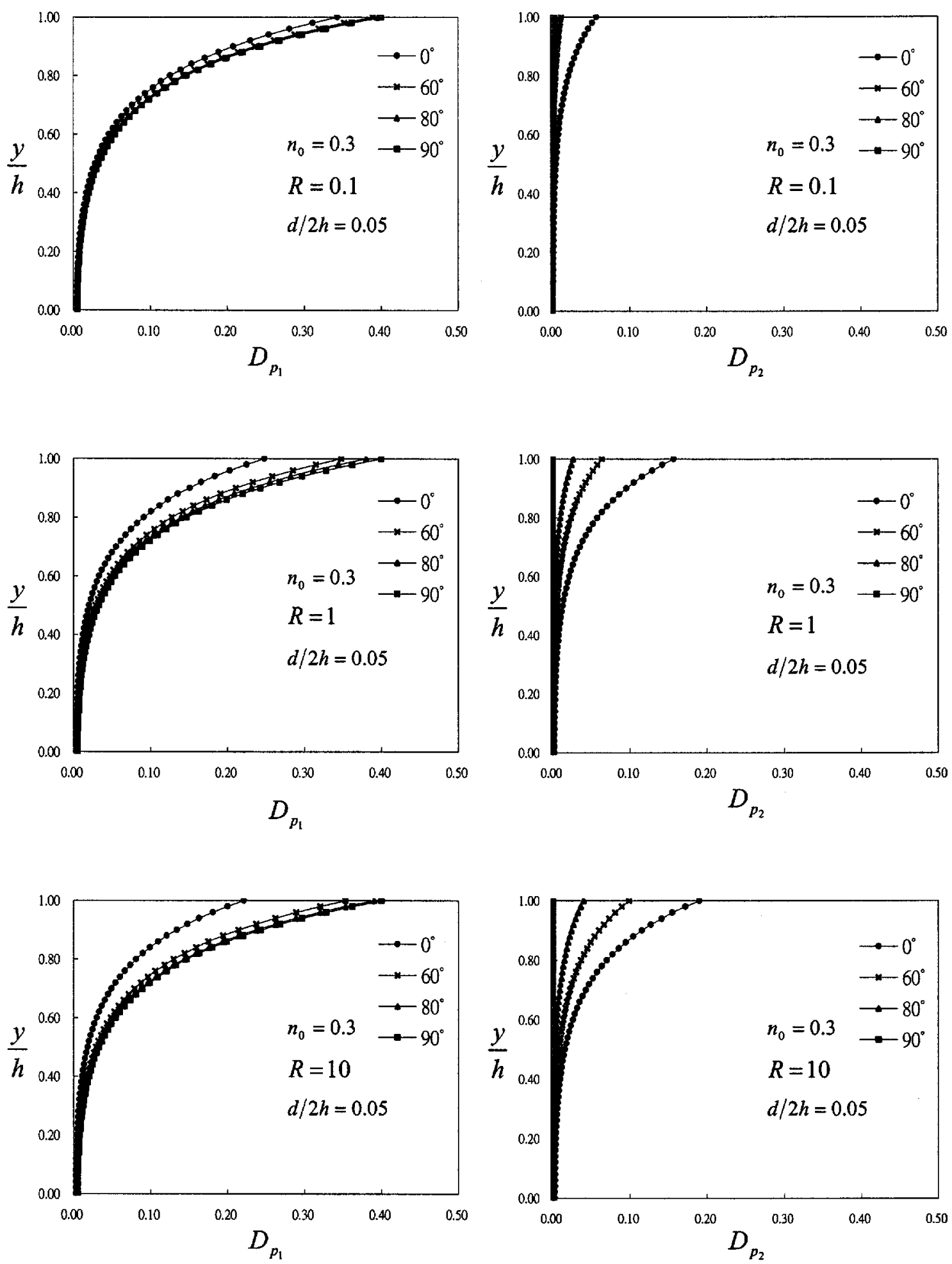

(a)

(b)

Fig. 4. Distributions of perturbed pressure amplitudes for thin porous wall at $x= \pm d / 2$ : (a) $D_{p_{1}}$; and (b) $D_{p_{2}}$

\section{Results}

In the present study, the so-called "thin" porous wall indicates that the thickness of the porous wall, compared to the wavelength, is extremely short. Hence, due to the property of the thin porous wall, the local phenomenon of surface of seepage [see Eqs. $(5 a)$, (5b), (10a), and (10b) as well as Muskat (1946)] is neglected.

In the following analysis, we only apply the porosity $n_{0}=0.3$ and the nondimensional wall thickness $d / 2 h=0.05$ for demonstration.

Fig. 4 reveals vertical distributions of perturbed pressure amplitudes $D_{p_{1}}$ and $D_{p_{2}}$ of homogeneous fluid on the interfaces of 

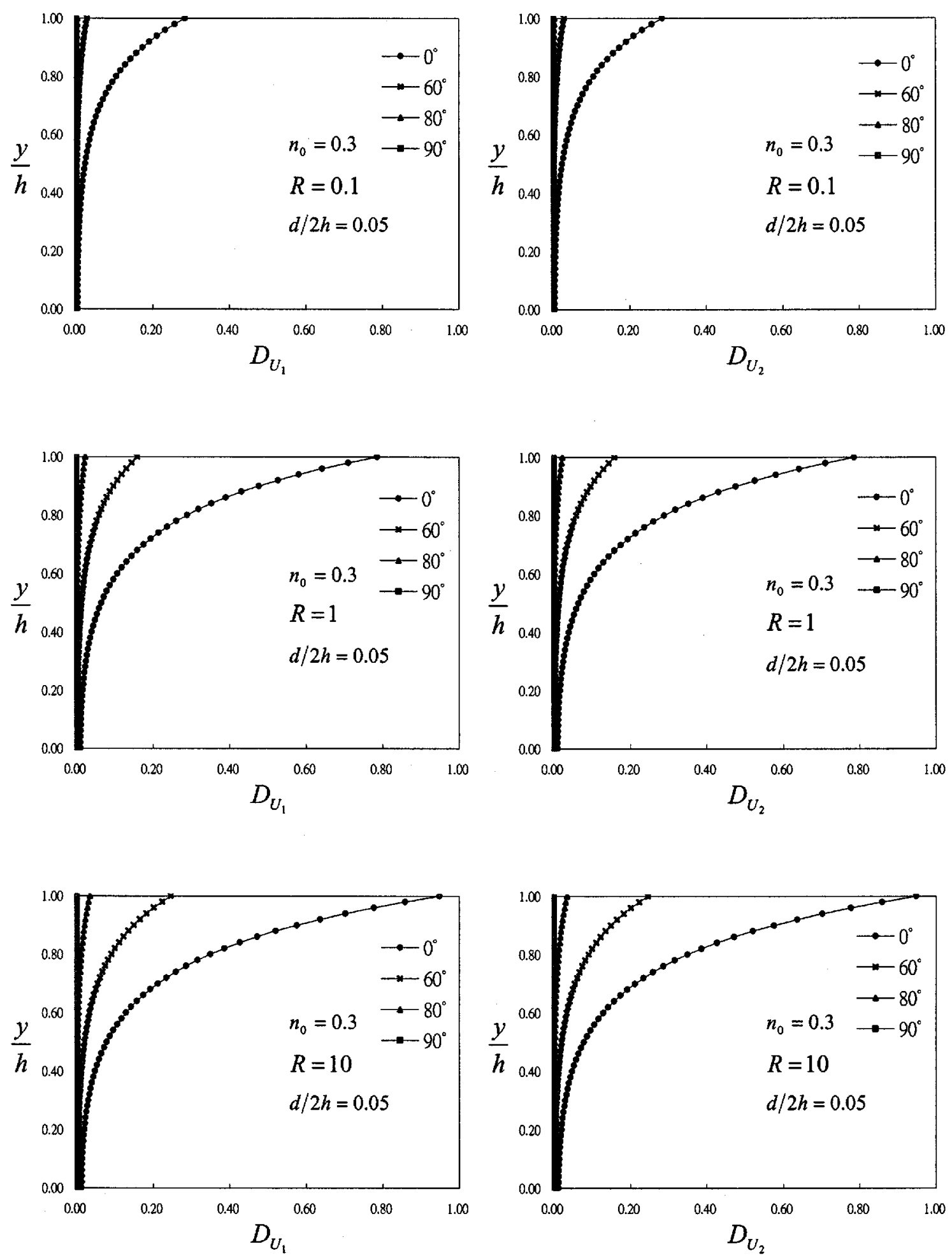

(a)

(b)

Fig. 5. Distributions of normal velocity amplitudes for thin porous wall at $x= \pm d / 2$ : (a) $D_{U_{1}}$; and (b) $D_{U_{2}}$

the thin porous wall $(x= \pm d / 2) . D_{p_{1}}$ and $D_{p_{2}}$ are given by Eqs. (41) and (46). Since it will be easier for the water wave to pass from Region (1) to Region (2) as the porous Reynolds number $R$ grows, it is observed that $D_{p_{2}}$ increases gradually when $R$ becomes larger. And as we expect, as the incident angle increases, $D_{p_{2}}$ decreases monotonically; when the incoming water wave is parallel to the porous wall (i.e., $\theta=90^{\circ}$ ), $D_{p_{2}}$ vanishes (i.e., when one side of a thin porous wall is affected by a parallel water wave, there is no water wave penetrating into the other side). This result agrees with our physical expectation when viscous effect of fluid on the wall is negligible.

Fig. 5 indicates vertical distributions of normal velocity am- 

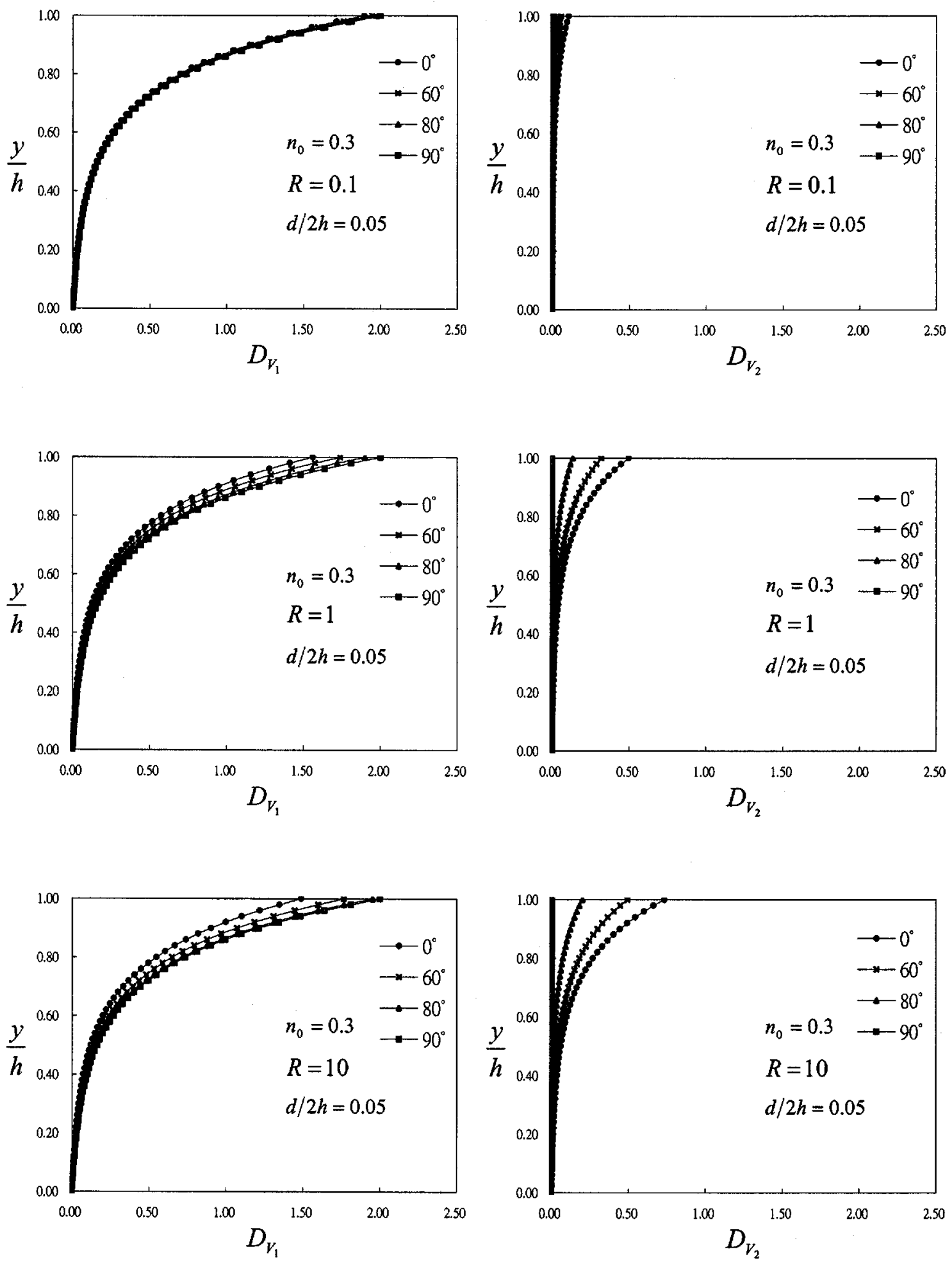

(a)

(b)

Fig. 6. Distributions of tangential velocity amplitudes for thin porous wall at $x= \pm d / 2$ : (a) $D_{V_{1}}$; and (b) $D_{V_{2}}$

plitudes $D_{U_{1}}$ and $D_{U_{2}}$ of homogeneous fluid on the thin porous wall $(x= \pm d / 2) . D_{U_{1}}$ and $D_{U_{2}}$ are given by Eqs. (51) and (56). It is found from Fig. 5(b) that $D_{U_{2}}$ shows a similar trend as $D_{p_{2}}$ does in Fig. 4(b) as $R$ and $\theta$ vary. It can be seen that when the incoming water wave is parallel to the porous wall (i.e., $\theta=90^{\circ}$ ), $D_{U_{2}}$, certainly disappears. Note that because of the mass flux continuity condition from the property of the thin porous wall, figures of $D_{U_{1}}$ are identical to those of $D_{U_{2}}$.

The vertical distributions of tangential velocity amplitudes $D_{V_{1}}$ and $D_{V_{2}}$ of homogeneous fluid are illustrated in Fig. 6. $D_{V_{1}}$ and $D_{V_{2}}$ are given by Eqs. (61) and (66). Beside same trend as Fig. 5, again, it is found that there is no tangential water wave penetrat- 

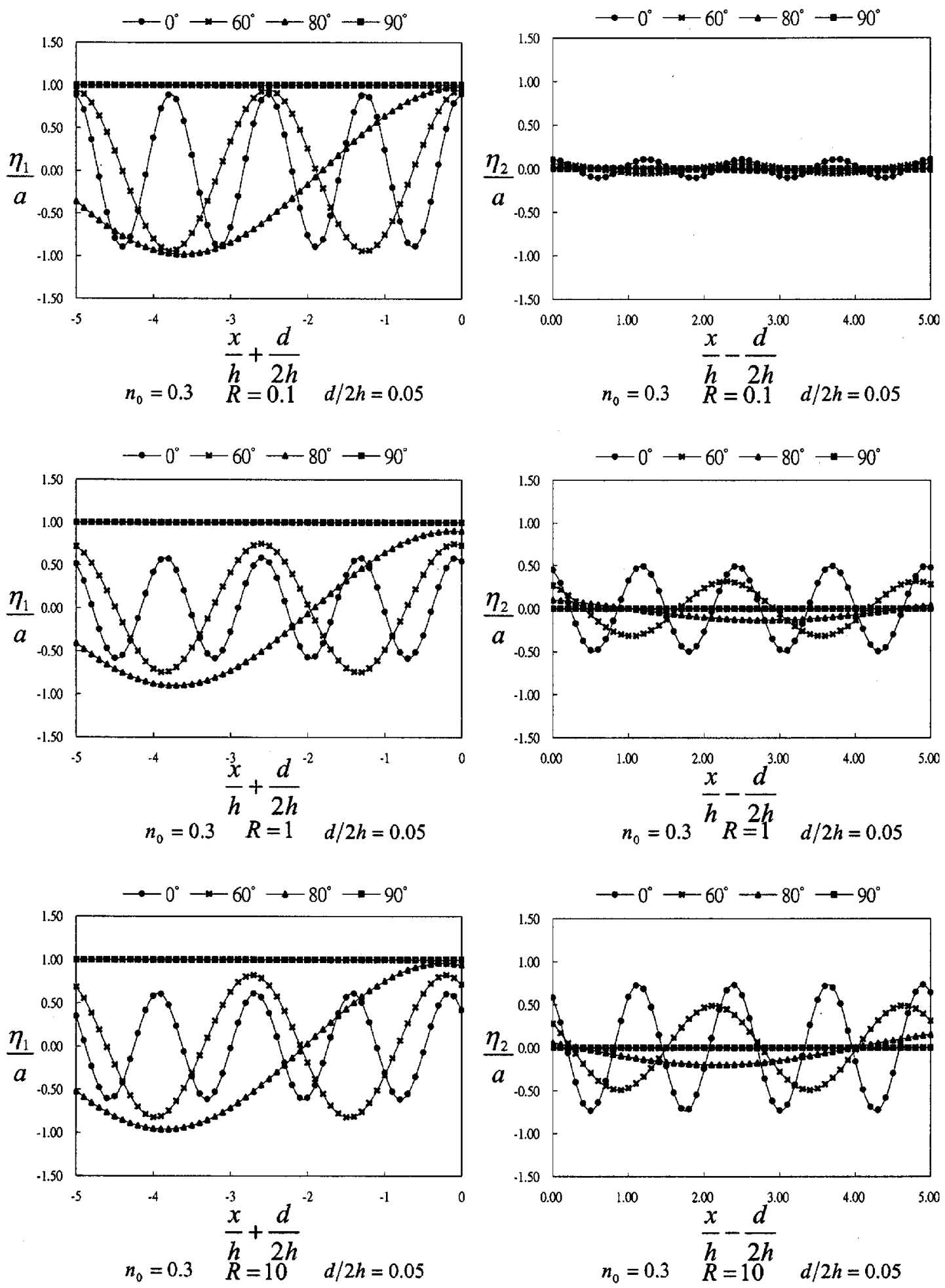

(a)

(b)

Fig. 7. Water wave profiles for thin porous wall: (a) $\eta_{1} / a$; and (b) $\eta_{2} / a$

ing into the original quiescent water field Region (2)] when the incoming water wave is parallel to the thin porous wall.

Fig. 7 presents the wave profiles in Regions (1) and (2). $\eta_{1} / a$ and $\eta_{2} / a$ are given by Eqs. (68) and (69). By analogy with above discussions, because it is easier for the water wave to pass from Region (1) to Region (2) as the porous Reynolds number $R$ grows, it is found that $\eta_{2} / a$ increases gradually when $R$ becomes larger. Besides, with the increase of the incident angle, $\eta_{2} / a$ decreases monotonically. And when the incoming water wave is parallel to the porous wall (i.e., $\left.\theta=90^{\circ}\right), \eta_{2} / a$ vanishes. This, again, confirms our physical expectation that when one side of a thin porous wall is affected by a parallel water wave, there is no 


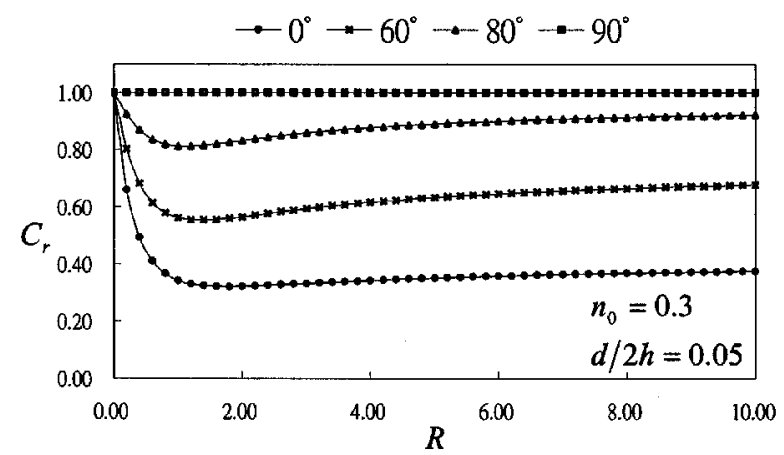

(a)

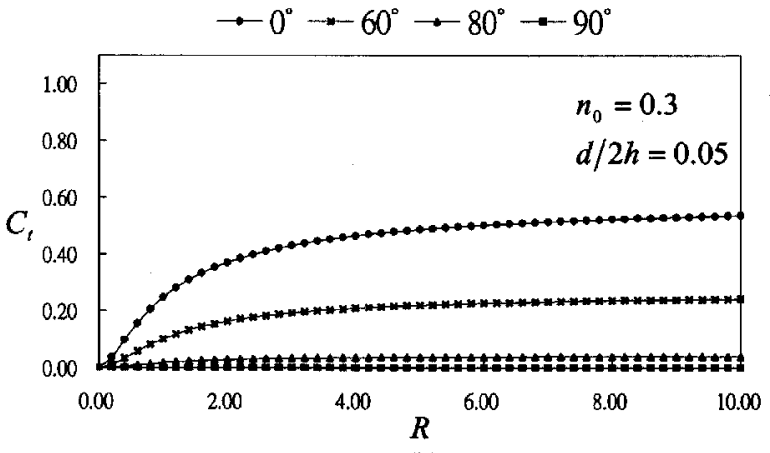

(b)

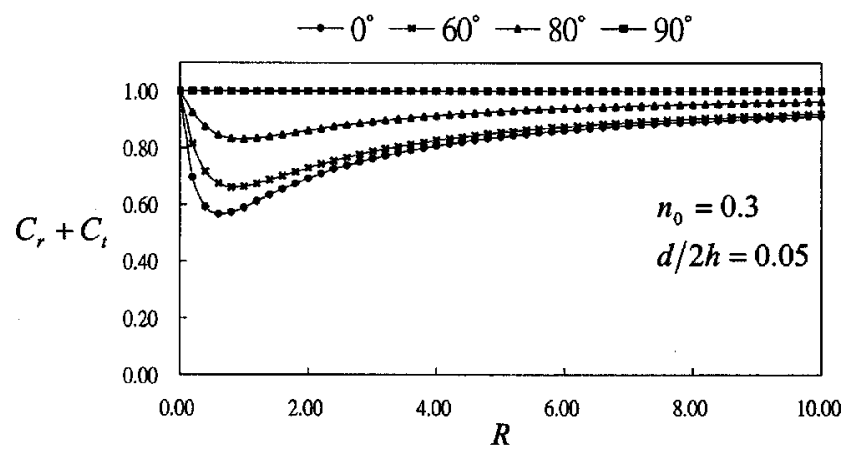

(c)

Fig. 8. Variations of reflection coefficients, transmission coefficients, and their combinations with respect to porous Reynolds number $R$ by using our partial-slipping boundary condition: (a) $C_{r}$; (b) $C_{t}$; and (c) $C_{r}+C_{t}$

water wave penetrating into the other side (provided that the viscous effect of water is negligible).

The reflection coefficient $C_{r}$ and transmission coefficient $C_{t}$ are most important in the analysis of the wave field. $C_{r}$ and $C_{t}$ are defined by Eqs. (70) and (71). Fig. 8 shows $C_{r}, C_{t}$, and their combination $C_{r}+C_{t}$ with respect to the variations of $R$. Note that there is a minimum value of $C_{r}+C_{t}$ when $R$ is closed to 1 and $\theta$ is small. Also notice that in general $C_{r}+C_{t}$ is less than 1 due to the damping effect of the porous wall except when there is no wave passing through the porous wall (i.e., $\theta=90^{\circ}$ ). Based on the definition of $C_{r}+C_{t}$, Fig. 8 indeed demonstrates the fact that the wave energy (excluding the incoming wave) plus the damping loss due to the thin porous wall is conserved at any incident angle.

The values of $C_{r}, C_{t}$, and $C_{r}+C_{t}$ with respect to the incident angle $\theta$ are presented in Fig. 9. Comparing to Fig. 8, Fig. 9 can be seen more distinctly how $C_{r}$ and $C_{t}$ change owing to the variations of $\theta$. It is observed that with the increase of the incident
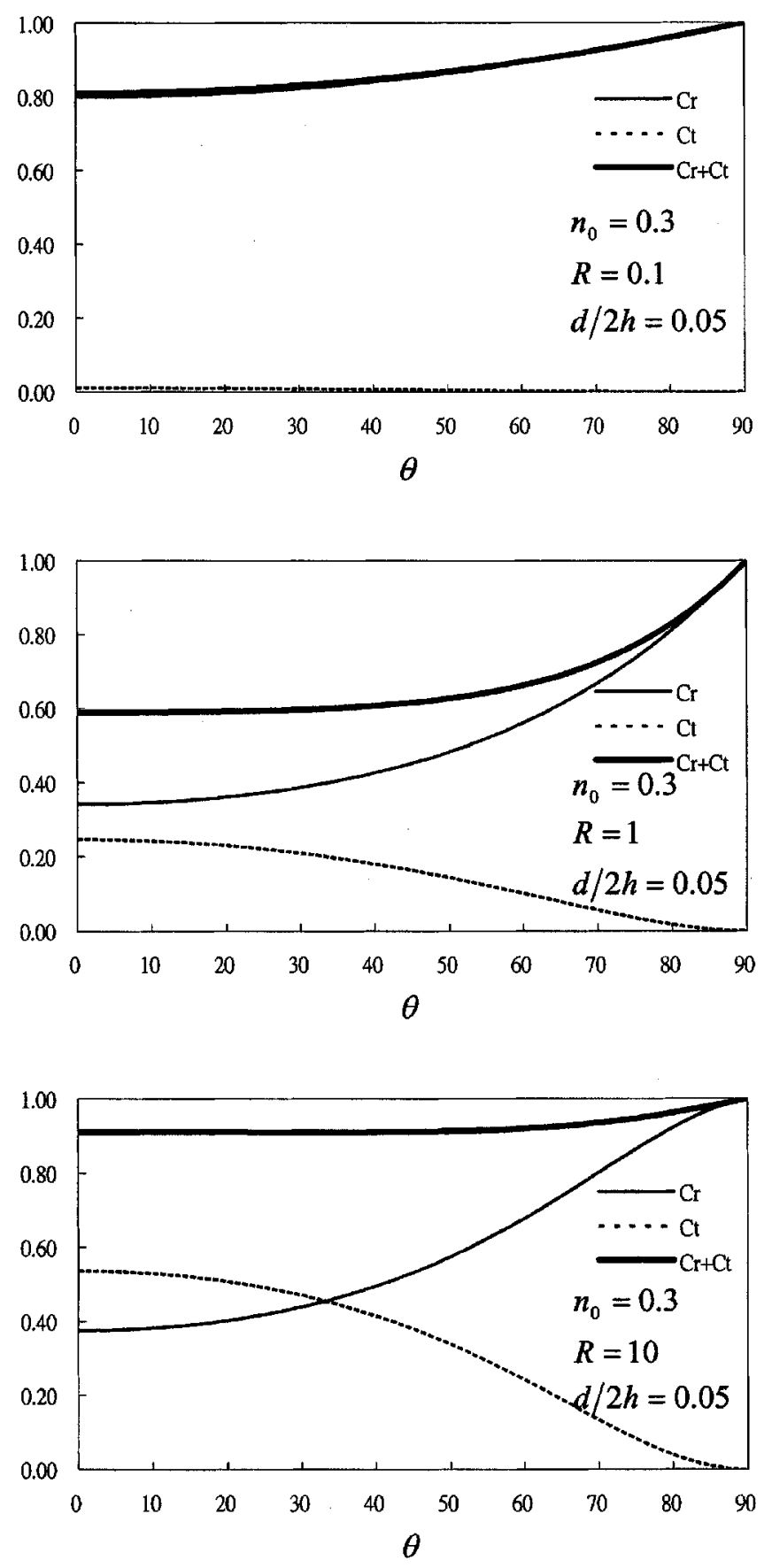

Fig. 9. Variations of reflection coefficients, transmission coefficients, and their combinations with respect to incident angle $\theta$ by using our partial-slipping boundary condition

angle, $C_{r}$ smoothly approaches to 1 , while $C_{t}$ approaches 0 simultaneously. On the other hand (see Fig. 10), with conventional slipping boundary condition applied by all the earlier studies, it is clearly found that $C_{r}$ and $C_{t}$ approach 0 and 1, respectively, with the increase of the incident angle; hence the transmitted wave side unreasonably captures all the wave energy as water wave is parallel to the porous wall. It obviously generates a paradoxical result. Therefore, it is concluded that our newly proposed partialslipping boundary condition really reveals the advantage of the present study compared to earlier ones. 

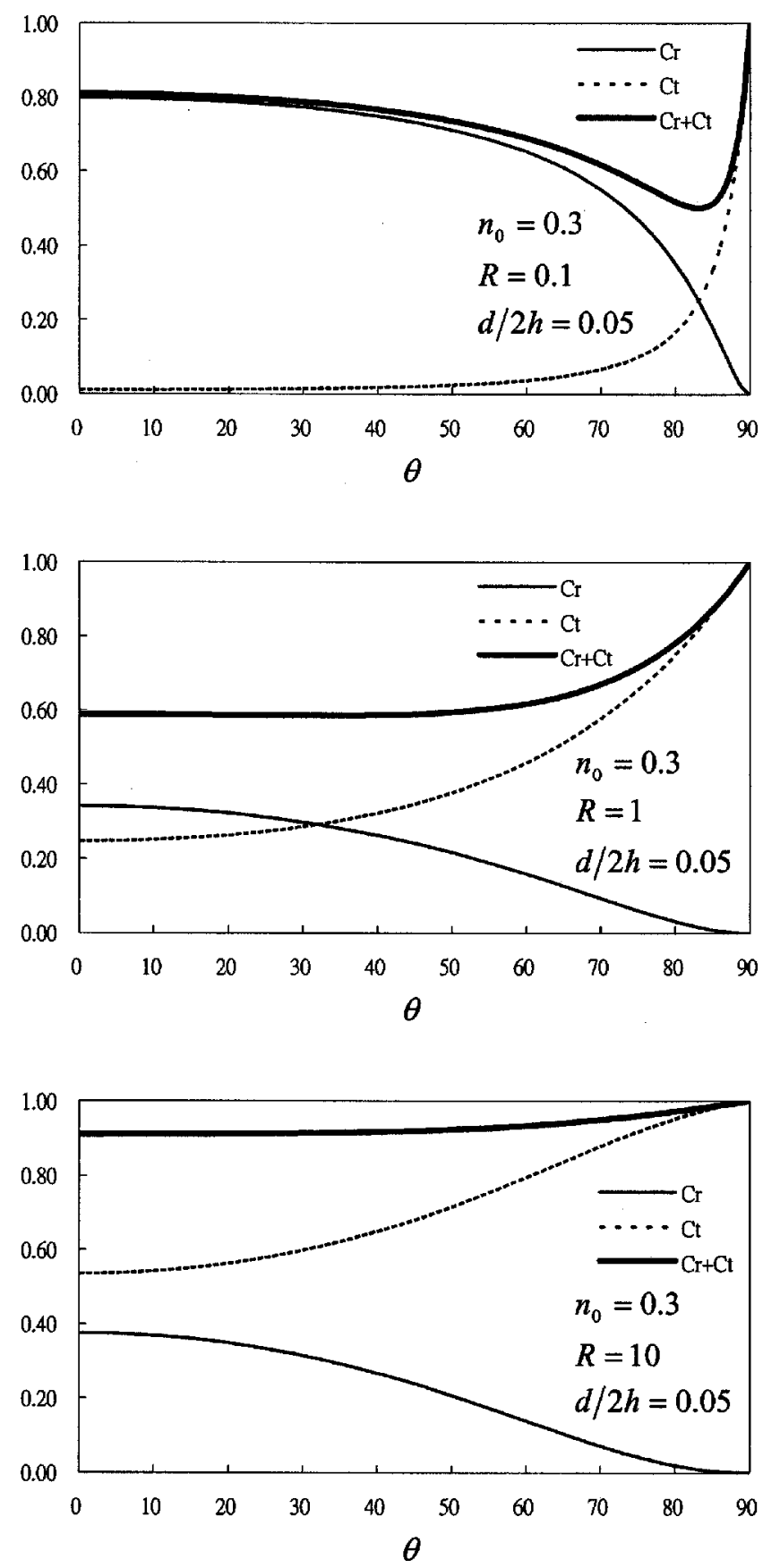

Fig. 10. Variations of reflection coefficients, transmission coefficients, and their combinations with respect to incident angle $\theta$ by using conventional slipping boundary condition

\section{Conclusions}

Based on the concept that the wave direction remains unchanged as it permeates into the thin porous medium, the present study thus derives a new boundary condition on the interfaces of the porous wall. According to this new boundary condition, an analytical solution of oblique water waves impacting on thin porous walls is obtained. The result eliminates the paradoxical phenomenon that the water wave permeates completely into the original quiescent water field when its incoming direction is parallel to the porous wall as earlier studies. And as the incident angle approaches $90^{\circ}$, the reflection coefficient and the transmission coef- ficient reasonably converge to 1 and 0 respectively, while, on the contrary, those in the earlier studies converge to 0 and 1 .

Since the thickness of porous structures are extremely small compared to the actual wavelength, a more reasonable wave trapping analysis of a harbor or coastal structure with the wave direction being taken into account can be expected by applying the concept of this study.

\section{Acknowledgement}

This study is supported by the National Science Council of R.O.C, under Grant No. NSC90-2611-E002-015.

\section{Notation}

The following symbols are used in this paper:

$C_{r}=$ reflection coefficient;

$C_{t}=$ transmission coefficient;

$D_{p_{j}}=$ nondimensional amplitude of perturbed pressure in region $j, j=1,2$;

$D_{U_{j}}=$ nondimensional amplitude of normal velocity in region $j, j=1,2$;

$D_{V_{j}}=$ nondimensional amplitude of tangential velocity in region $j, j=1,2$;

$d=$ thickness of porous wall;

$g=$ gravitational acceleration;

$h=$ undisturbed water depth;

$k=$ specific permeability of porous medium;

$k_{0}=$ wave number of propagating wave;

$n_{0}=$ porosity of porous medium;

$p_{j}=$ perturbed pressure in region $j, j=1,2,3$;

$\mathrm{R}=$ porous Reynolds number;

$U_{j}=$ normal velocity in region $j, j=1,2$;

$V_{j}=$ tangential velocity in region $j, j=1,2$;

$\zeta=$ modified wave number of a propagating wave, $\zeta$ $=k_{0} \sin \theta$;

$\eta_{0}=$ wave profile of an incoming wave;

$\eta_{1}=$ wave profile in Region (1) with the incoming wave being excluded;

$\eta_{2}=$ wave profile in Region (2);

$\theta=$ angle of incidence;

$\mu=$ dynamic viscosity of fluid;

$\xi=$ modified wave number of propagating wave, $\xi=k_{0} \cos \theta$;

$\rho=$ density of fluid;

$\Phi_{j}=$ velocity potential of flow in region $j, j=1,2,3$;

$\phi_{j}=$ velocity potential of flow in region $j, j=1,2,3 ; \Phi_{j}$ $=(x, y, z, t)=\mathrm{R}\left[\phi_{j}(x, y) e^{i\left(k_{0} \sin \theta z-\omega t\right)}\right]$; and

$\omega=$ angular frequency of the water wave.

\section{References}

Biot, M. A. (1962). "Mechanics of deformation and acoustic propagation in porous media." J. Appl. Phys., 33, 1482-1498.

Chwang, A. T. (1983). "A porous wave-maker theory." J. Fluid Mech., 132, 395-406.

Chwang, A. T., and Dong, Z. (1984). "Wave trapping due to a porous plate." Proc., 15th Symp. on Naval Hydrodynamics, 407-417.

Chwang, A. T., and Li. W. (1983). "A piston-type porous wave-maker theory." J. Eng. Math., 17, 301-313.

Dalrymple, R. A., Losada, M. A, and Martin, P. A. (1991). "Reflection 
and transmission from porous structures under oblique wave attack." J. Fluid Mech., 224, 625-644.

Huang, L. H. (1991). "The inertial effect of a finite thickness porous wave-maker." J. Hydraul. Res., 29, 417-432.

Huang, L. H., Hsieh, P. C., and Chang, G. Z. (1993). "Study on porous wave makers." J. Eng. Mech., 119(8), 1600-1614.

Madsen, O. S. (1974). "Wave transmission through porous structures." $J$. Waterw., Harbors Coastal Eng. Div., Am. Soc. Civ. Eng., 100(3), 169188.

Muskat, M. (1946). The flow of homogeneous fluids through porous media, Edwards, Ann Arbor, Mich.
Sahoo, T., Chan, A. T., and Chwang, A. T. (2000). "Scattering of oblique surface waves by permeable barriers." J. Waterw., Port, Coastal, Ocean Eng., 126(4), 196-205.

Sollitt, C. K., and Cross, R. H. (1972). "Wave transmission through permeable breakwaters." Proc., 13th Coastal Engineering Conf., ASCE, New York, Vol. 3, 1827-1846.

Taylor, G. I. (1956). "Fluid flow in regions bounded by porous surfaces." Proc. R. Soc. London, Ser. A, 234, 456-475.

Tyvand, P. A. (1986). "Long waves in a canal with a porous plate located at a step." Phys. Fluids, 29(7), 2027-2028. 